\title{
EVALUACIÓN DE LA CALIDAD DE VIDA, ESTADO EMOCIONAL Y ESTRATEGIAS DE AFRONTAMIENTO EN PACIENTES CON ENFERMEDAD NEOPLÁSICA PULMONAR
}

\section{ASSESSMENT OF QUALITY OF LIFE, EMOTIONAL STATE, AND COPING SKILLS IN PATIENTS WITH NEOPLASTIC PULMONARY DISEASE}

\author{
Raquel Rodríguez-Quintana', Florentino Hernando-Trancho², Juan Antonio Cruzado3, \\ $M^{a}$ Eugenia Olivares ${ }^{2}$, Ismael Jamal ${ }^{4}$ y Pilar Laserna ${ }^{3}$ \\ ' Hospital Sant Joan de Déu, Palma de Mallorca. Obra Social "La Caixa" \\ 2 Hospital Clínico San Carlos de Madrid \\ 3 Universidad Complutense de Madrid \\ ${ }^{4}$ Hospital San Rafael de los Hermanos de San Juan de Dios, Madrid. Obra Social "La Caixa"
}

Resumen

Objetivo: evaluar la calidad de vida, el estado emocional (ansiedad y depresión) y las estrategias de afrontamiento de pacientes con cáncer de pulmón antes de ser intervenidos quirúrgicamente.

Método: 121 pacientes ambulatorios con diagnóstico de cáncer de pulmón no microcítico (CPNMC) fueron entrevistados antes de la cirugía. Los cuestionarios empleados fueron el EORTC QLQ-C30 y el módulo específico de pulmón LC13, la escala de Ansiedad y Depresión Hospitalaria (HAD) y el cuestionario de Ajuste mental al Cáncer (MAC). Datos demográficos y clínicos también fueron recogidos. Comparamos grupos en relación al sexo y al tipo de intervención quirúrgica.

Resultado: La calidad de vida global del paciente con cáncer de pulmón que va a ser intervenido fue aceptable $(72,5)$. La mayoría de pacientes presentaron altos niveles de funcionamiento y bajos niveles de sintomatología ambos medidos por los cuestionarios EORTC (QLQ-C30 y LC13). Sólo los neumonectomizados tuvieron más déficit en calidad de vida presentando peor funcionamiento de rol, más
Abstract

Purpose: To assess the quality of life, emotional state (anxiety and depression) and coping skills of a sample of lung cancer patients before the surgery.

Methodology: 121 ambulatory patients with non small-cell lung cancer (NSCLC) were interviewed before the surgery. Patients filled the EORTC QILQ-C30 questionnaire, and the EORTC-LC13 lung module, the Hospital Anxiety and Depression Scale (HADS) and the Mental Adjustment to Cancer (MAC) Scale. Demographic and clinical data were also recorded. We compared the groups according to the gender and to the type of surgery treatment.

Result: The quality of life scores of the patients before the surgery were acceptable (72.5). On average patients had high levels of functioning and low levels of symptoms in both EORTC questionnaries (QLQ-C30 and the lung cancer specific module LC13). Only pneumonectomy patients have limitations in some quality of life areas (role functioning, pain in the chest and haemoptysis). Few differences were observed between the genders. Dyspnoea

Correspondencia:

Raquel Rodríguez Quintana

Equipo de Atención Psicosocial (EAPS) del Hospital Sant Joan de Déu de Palma de Mallorca

C/ Passeig de Cala Gamba, 35

07007 Palma de Mallorca

E-mail: rrodriguezquintana@ohsjd.es 
dolor en el pecho y más sangrado. Se encontraron diferencias en calidad de vida según la variable sexo. La disnea fue el principal síntoma que se asoció a déficit en calidad de vida, depresión y ansiedad, además de otros síntomas físicos (fatiga y dolor). El $20,53 \%$ presentó sintomatología depresiva y el $32,15 \%$ ansiosa. Las estrategias de afrontamiento más utilizadas por los pacientes con cáncer de pulmón fueron fatalismo $(71,67 \%)$ y espíritu de lucha $(70,49 \%)$.

Conclusiones: investigar la influencia de los factores emocionales en el pre-operatorio puede ser útil para identificar los factores de riesgo y las dificultades en la adaptación a la enfermedad y así poder diseñar intervenciones psicológicas. Futuras investigaciones y estudios longitudinales son necesarios para establecer comparaciones entre grupos (pre y post-operatorio).

Palabras clave: Calidad de vida, ansiedad, depresión, afrontamiento y cáncer de pulmón.

\section{INTRODUCCIÓN}

El cáncer de pulmón representa aproximadamente el $12 \%$ de todos los diagnósticos de cáncer, convirtiéndose en el tumor maligno más frecuente en países desarrollados. En el $85 \%$ de los casos se diagnostica en fases avanzadas, presentando síntomas característicos como disnea, fatiga, dolor torácico, tos, disfonía, disfagia, anorexia, pérdida de peso e insomnio. En etapas tempranas es una enfermedad que no presenta muchos síntomas pero si se encuentra en etapas avanzadas puede afectar a la calidad de vida de la persona que lo padece ${ }^{(1)}$.

Según la Organización Mundial de la Salud, la calidad de vida se define como "percepción que un individuo tiene de su lugar en la existencia, en el contexto de la cultura y del sistema de valores en los que vive y en relación con sus expectativas, sus normas, sus inquietudes. Se trata de un concepto muy amplio que está influido de modo complejo por la salud física del was the main symptom related to low quality of life, depression, anxiety and some physical symptoms (fatigue and pain). $20.53 \%$ displayed high level of depressive symptoms and $32.15 \%$ anxiety. The most prominents ways of coping used by lung cancer patients are fatalism (71.67\%) and fighting spirit (70.49\%).

Conclusions: Investigating the influence of psychological factors on the preoperative could be very useful for an early identification of psychological risk factors and bad adjustment to the illness and be able to plan a psychological intervention. Further investigations and longitudinal studies are needed especially to establish comparative among pre and post-operative moments.

Keywords: Quality of life, anxiety, depression, coping skills and lung cancer.

sujeto, su estado psicológico, su nivel de independencia, sus relaciones sociales, así como su relación con los elementos esenciales de su entorno". Esta definición de calidad de vida hace referencia a población general pero en el caso de pacientes oncológicos debiéramos incluir los efectos de la enfermedad y los tratamientos.

Aaronson es uno de los autores dedicados a temas de calidad de vida en población oncológica. Las dimensiones que él propone son las siguientes ${ }^{(2)}$ :

- Estado funcional

- Síntomas físicos relacionados con la enfermedad y el tratamiento

- Función psicológica

- Función social y familiar

Posteriormente se añaden los siguientes subcomponentes para evaluar la calidad de vida del paciente oncológico ${ }^{(3)}$ :

- Función física: realización de actividades de autocuidado, el estado funcional, la movilidad, la actividad física y la función de rol, el trabajo y las tareas domésticas. 
- Síntomas relacionados con la enfermedad y el tratamiento: dolor, la disnea, las náuseas, la caída del pelo y la sedación

- Función psicológica: ansiedad y la depresión

- Función social: interrupción de las actividades sociales normales

- Dimensión espiritual o existencial

- Satisfacción con los cuidados médi$\cos$

La evaluación de la calidad de vida ha empezado a ser un punto de partida para evaluar los resultados de los tratamientos a corto y largo plazo y es utilizado como un factor predictor del pronóstico de supervivencia en el cáncer de pulmón ${ }^{(4-12)}$.

Los pacientes operados de cáncer de pulmón puntúan peor su calidad de vida, experimentan más problemas físicos y psicosociales que los intervenidos de colon y de próstata ${ }^{(3)}$.

Hay diferencias en calidad de vida en pacientes de cáncer de pulmón dependiendo de la intervención quirúrgica. Datos preoperatorios en pacientes candidatos a una resección pulmonar muestran que las escalas de calidad de vida más afectadas son la escala física y la emocional (limitaciones de rol debidos a problemas físicos y emocionales, limitaciones en el funcionamiento social, percepción de salud general y salud mental). Estas escalas permanecen por debajo de los parámetros de población general incluso 3 meses después de la intervención quirúrgica ${ }^{(13)}$.

La neumonectomía, que implica la extirpación de un pulmón entero, produce reducción en el funcionamiento pulmonar y se asocia con peor calidad de vida ${ }^{(12,14,15)}$.

Ante un evento vital estresante, como es el diagnóstico de cáncer, la persona realiza una interpretación y evaluación de la situación. Si la persona interpreta la situación como una amenaza de la cual carece de recursos para hacerle para controlar las consecuencias negativas, se generará una reacción emocional caracterizada por la ansiedad. En cambio, si la persona valora la situación como una pérdida importante (de salud, de funcionalidad, de esperanza de vida...) generará una reacción emocional de tristeza que puede desencadenar en una depresión ${ }^{(16)}$.

La mayoría de investigaciones sugieren que la prevalencia de trastornos psicopatológicos en pacientes oncológicos se sitúa entre el 25-50\%, variando la cifra según la metodología empleada y los criterios diagnósticos utilizados ${ }^{(17)}$. La prevalencia de sintomatología depresiva ante un diagnóstico de cáncer de pulmón se estima entre un $15 \%$ y $44 \%{ }^{(9,18)}$. En un estudio, un tercio de los pacientes con cáncer de pulmón presentaba depresión antes de la cirugía, y persistió en más del 50\% de éstos después de que la cirugía tuviera lugar ${ }^{(18)}$. Los estudios incluso reportan que algunos pacientes presentan depresión después de un tratamiento curativo, en este caso la resección quirúrgica pero es menor que en el preoperatorio ${ }^{(19,20)}$. En el estudio de Marc et al. refieren que pacientes con bajas puntuaciones en estado funcional presentan mayor sintomatología ansiosa y depresiva $^{(21)}$.

En los pacientes con cáncer de pulmón, la literatura científica postula que ansiedad moderada a severa está presente en el 10$45 \%$ de los pacientes ${ }^{(9,18,22,23)}$.

Las tres necesidades que consideraron básicas en la muestra del estudio de Liao et al. fueron: 1) sistema de salud e información, 2) psicológicas y 3) cuidado y apoyo al paciente ${ }^{(24)}$. En un estudio se entrevistó a 103 pacientes diagnosticados de cáncer de pulmón donde les preguntaron acerca de sus necesidades. El 51,4\% iban encaminados a servicios relacionados con la asistencia psicológica ${ }^{(25)}$.

Tanto la ansiedad como la depresión tienen un gran impacto en la calidad de vida de los pacientes oncológicos ${ }^{(9,26,27)}$. Cody et al. sugieren que los trastornos 
psiquiátricos en pacientes con cáncer de pulmón está significativamente asociado a un empeoramiento en la dimensión de calidad de vida referida a funcionamiento social ${ }^{(28)}$. Disnea y sintomatología depresiva correlacionó con déficits en funcionamiento físico y emocional en una muestra de mujeres con cáncer de pulmón ${ }^{(29)}$.

Existe correlación negativa entre depresión y calidad de vida global y con la subescala función cognitiva, correlacionando de manera positiva depresión y la subescala dolor mientras que la ansiedad afecta particularmente en funcionamiento social, funcionamiento físico, sintomatología física (disnea, toser, hemoptisis) y psicológica (baja autoestima y sentimiento de que la vida no tiene sentido) ${ }^{(22,30,31)}$.

A la hora de afrontar el diagnóstico de una enfermedad oncológica se hace referencia a respuestas cognitivas y conductuales de los pacientes ante el cáncer, comprendiendo la valoración (significado del cáncer para el sujeto) y las reacciones subsiguientes (lo que el individuo piensa y hace para reducir la amenaza que supone el cáncer) ${ }^{(32)}$.

Moorey y Greer plantean un modelo de funcionamiento del enfermo ante el cáncer denominado "esquema de supervivencia" entendido como la capacidad de adaptación, la respuesta emocional y los estilos de afrontamiento que ponen en marcha los pacientes oncológicos a la hora de conocer su diagnóstico o el estado de la enfermedad. En el esquema de supervivencia propone una evaluación cognitiva por la cual el sujeto considera tres factores ${ }^{(33)}$ :

1. La visión del diagnóstico que "¿qué amenaza supone mi enfermedad?"

2. El tipo de control que ejerce sobre la enfermedad " ¿qué puedo hacer yo para enfrentarme a la enfermedad"

3. A visión del pronóstico “¿cuál es el pronóstico de mi enfermedad?"

En respuesta a estos 3 factores, los auto- res plantean 5 estrategias de afrontamiento en los pacientes con cáncer:

- Espíritu de lucha

- Evitación/negación

- Indefensión/desesperanza

- Preocupación ansiosa

- Fatalismo

El tipo de afrontamiento determinará la morbilidad psicológica de los pacientes. Los pacientes que muestran niveles menores de espíritu de lucha y mayores niveles en desesperanza y preocupación ansiosa presentan peor adaptación a la enfermedad (entendida como una mayor presencia de depresión y ansiedad) y una peor calidad de vida(34). No cabe duda de que el espíritu de lucha es la estrategia más favorable de cara a una buena adaptación a la enfermedad y es la que correlaciona con mejor pronóstico.

Resultan curiosos los datos aportados por el estudio de Vos et al. donde afirman que pacientes con altos o moderados niveles de negación reportan mejor funcionamientos social menor ansiedad y depresión que pacientes con nivel bajo de negación. Parece ser que en los pacientes con cáncer de pulmón la negación pueden tener un efecto protector ${ }^{(35) .}$

Los pacientes con cáncer de pulmón presentan respuestas cognitivas diferentes a los pacientes con otros tipos de cáncer, atribuyéndose los primeros la culpa de la enfermedad por su voluntad de fumar, ayudándoles a bloquear sus recursos personales para desarrollar estrategias de afrontamiento y adaptación ${ }^{(36)}$.

\section{OBJETIVOS}

Los objetivos generales fueron: determinar los niveles de calidad de vida, la sintomatología ansiosa y/o depresiva en los pacientes con NSCLC (Non Small Cell Lung Cancer) intervenidos quirúrgicamente en el Servicio de Cirugía Torácica del Hospital Universitario Clínico San Carlos. Estudio de 
la relación entre la calidad de vida y el estado emocional en pacientes prequirúrgicos y las diferencias según el tipo de cirugía y sexo. Por último, evaluación de las estrategias de afrontamiento que llevan a cabo en situación de enfermedad crónica.

Los objetivos específicos fueron:

- Obtener datos descriptivos de los pacientes de cáncer de pulmón en torno a las dimensiones de calidad de vida (escalas funcionales, sintomáticas, calidad de vida global, síntomas adicionales padecidos por los pacientes de cáncer, impacto económico, síntomas específicos del cáncer de pulmón y efectos secundarios de la quimioterapia y radioterapia)

- Comparar las puntuaciones de calidad de vida con datos normativos para muestra europea.

- Determinar Prevalencia de sintomatología depresiva y ansiosa

- Describir las estrategias de afrontamiento que atizan los pacientes

- Estudiar la relación entre calidad de vida con la sintomatología emocional

- Determinar el efecto de la variable clínica "intervención quirúrgica" y "sexo" en las variables calidad de vida y estado emocional

- Estudiar la relación entre calidad de vida y estado emocional

- Estudiar de la relación entre calidad de vida y estrategias de afrontamiento

- Estudio de la relación entre estado emocional y estrategias de afrontamiento

\section{MÉTODO}

\section{MUESTRA}

La muestra estuvo formada por pacientes con NSCLC intervenidos quirúrgicamente en del Servicio de Cirugía Torácica del Hospital Universitario Clínico San Car- los de Madrid. Se incluyeron todos aquellos que de manera voluntaria aceptaron participar en la investigación y que cumplían criterios de inclusión. Los participantes fueron entrevistados entre los meses de octubre de 2007 a mayo de 2009.

Los criterios de inclusión para la investigación son los siguientes:

- Mayor de 18 años

- Sospecha inicial de diagnóstico de enfermedad neoplásica pulmonar y ser subsidiario de intervención quirúrgica.

- Recibir o haber recibido tratamiento quirúrgico (resección, lobectomía, neumonectomía u otros).

- Sin evidencia de metástasis a distancia en la actualidad.

- Perteneciente al Área de Salud 7 de Madrid.

Los criterios de exclusión para la investigación son los siguientes:

- Otros diagnósticos de enfermedad pulmonar

- Recibir tratamiento oncológico activo en el momento de la investigación

- Deterioro cognitivo que les imposibilite responder los cuestionarios

- Presencia de psicopatología severa

\section{DISEÑO}

Las variables independientes de esta investigación fueron:

1. Tipo de intervención quirúrgica (resección segmentaria, lobectomía, neumonectomía y otros tipos de cirugía)

\section{Sexo}

Las variables dependientes $\mathrm{u}$ los instrumentos de evaluación fueron los siguientes:

- Sintomatología depresiva medida con la Escala de Depresión y Ansiedad 
Hospitalaria (7 items, HADS-D) $)^{(37,38)}$.

- Sintomatología ansiosa medida con la Escala de Depresión y Ansiedad Hospitalaria (7 items, HADS-A) ${ }^{(37,38)}$

- Cuestionario de Calidad de Vida EORTC QLQ-C30(2,39). Es un cuestioario de 30 ítems), compuesto de 5 subescalas funcionales: física, laboral, cognitiva, emocional y social; 3 subescalas sintomáticas: fatiga, dolor, náuseas y vómitos; 1 subescala de calidad de vida global (salud y calidad de vida); y síntomas adicionales padecidos por los pacientes de cáncer: disnea, pérdida de apetito, trastornos del sueño, estreñimiento y diarrea, e Impacto económico observado que se deriva de la enfermedad y su tratamiento.

- Cuestionario de Calidad de Vida en su módulo específico para cáncer de pulmón EORTC LC13 ${ }^{(40,41)}$. Esta escala mide los Síntomas específicos del cáncer de pulmón: escalas de disnea y dolor, ítems de hemoptisis, tos. Así como los efectos secundarios de la quimioterapia y radioterapia: ítems de pérdida de pelo, neuropatía, dolor en la boca y disfagia.

- Estrategias de afrontamiento medidas con el cuestionario Cuestionario de Ajuste Mental al Cáncer (MAC, 40 ítems) $)^{(42-43)}$. Consta de 40 ítems que evalúa las estrategias de afrontamiento: espíritu de lucha, desesperanza/indefensión, evitación, fatalismo y preocupación ansiosa.

Las variables moduladoras fueron:

1. variables sociodemográficas (nivel de estudios, profesión, situación laboral y estado civil).

2. variables médicas (tratamiento antineoplásico recibido, enfermedades médicas previas, antecedentes psicopatológicos previos, tratamiento psicofarmacológico, tratamiento psicológico/psiquiátrico, fumador/no fumador, consumo alcohol y consumo de otras sustancias)

\section{PROCEDIMIENTO}

En primer lugar se procedió a reclutar a la muestra de personas voluntarias que participaron en el estudio. Las personas citadas acudieron de forma individual a la evaluación que se realizó de forma ambulatoria. Antes de la administración de los cuestionarios se explicaron los objetivos de la investigación de manera clara y comprensible, la participación anónima y voluntaria, la posibilidad de rechazar participar en el estudio sin que ello le perjudicara de ninguna forma y se asegura la absoluta confidencialidad de los datos aportados. Si comprendía todos estos puntos procedía a firmar un consentimiento informado. A continuación se recogieron los datos sociodemográficos, clínicos y psicosociales del paciente y posteriormente se pasó a cumplimentar los cuestionarios. El tiempo de evaluación no superó los 90 minutos.

\section{RESULTADOS}

Los datos fueron procesados por el paquete estadístico SPSS (Stadistical Package for Social Sciences, versión 17.0) para Windows.

\section{Descriptivos Muestra prequirúrgica $(\mathrm{n}=121)$}

Los datos referentes a las variables sociodemográficas y clínicas se muestran en las tablas 1 y 2 respectivamente.

El perfil de paciente fue de hombre (62\%), edad media 63 años (DT $\pm 10,85)$, casados $(62,3 \%)$, con estudios primarios 
$(55,7 \%)$, la mayoría jubilados $(54,9)$ que han trabajado por cuenta ajena (59\%).

El tipo de intervención quirúrgica predominante ha sido la resección segmentaria $(39,3 \%)$ y la lobectomía $(35,2)$. El estadio es más variable, siendo el IB (18,9\%) y el IIIA $(9,8 \%)$ los más frecuentes en nuestra investigación. Más de la mitad de los pacientes no habían tenido antecedentes personales de cáncer $(49,2 \%)$. La mitad de ellos eran fumadores previos (64,8\%). La mayoría de los pacientes no presentaban antecedentes psicológicos $(74,6 \%)$ ni habían recibido psicofármacos $(70,5 \%)$.

Los datos de calidad de vida se exponen en la tabla 3. Para interpretar los da- tos debemos tener en cuenta que a mayor puntuación en las escalas de funcionales mejor calidad de vida y a mayor puntuación en las escalas de síntomas indican mayor sintomatología y peor calidad de vida.

\section{Estado emocional}

En las tabla 4 y podemos ver reflejado la media y la desviación típica referente a sintomatología ansiosa y/o depresiva de nuestra muestra, así como los casos en ekl rango normal, subclínicos y clínicos. El $4,46 \%$ de los pacientes presentaron altos niveles de depresión y al $11,61 \%$ de ansiedad.

\section{Tabla 1. Datos sociodemográficos de la muestra prequirúrgica.}

\begin{tabular}{|c|r|}
\hline \multicolumn{1}{|c|}{ DATOS } & \\
\hline SEXO & Hombre $76(62,3 \%)$ \\
& Mujer $45(36,9 \%)$ \\
\hline EDAD & Media: 63,18 \\
& D.T: 10,856 \\
& Mínimo 23 \\
& Máximo 81 \\
\hline ESTADO CIVIL & Casado $76(62,3 \%)$ \\
& Soltero $14(11,5 \%)$ \\
& Viudo $13(10,7 \%)$ \\
& Divorciado $6(4,9 \%)$ \\
Pareja $3(2,5 \%)$ \\
\hline PROFESIÓN & Trabajo por cuenta ajena $72(59,0 \%)$ \\
& Ama de casa $15(12,3 \%)$ \\
& Funcionario $15(12,3 \%)$ \\
& Trabajo por cuenta propia $12(9,8 \%)$ \\
\hline SITUACIÓN & Jubilado $67(54,9 \%)$ \\
& Ama de casa $17(13,9 \%)$ \\
& Activo $13(10,7 \%)$ \\
& Baja $12(9,8) \%$ \\
& Paro $4(3,3)$ \\
\hline NIVEL DE ESTUDIOS & Primarios $68(55,7)$ \\
& Sundarios $30(24,6 \%)$ \\
& Superiores $18(14,8 \%)$ \\
\hline
\end{tabular}


Tabla 2. Datos clínicos de la muestra prequirúrgica.

\begin{tabular}{|c|c|}
\hline DATOS & \\
\hline TIPO DE CIRUGÍA & $\begin{array}{r}\text { Resección segmentaria } 48(39,3 \%) \\
\text { Lobectomía } 43(35,2 \%) \\
\text { Neumonectomía } 11(9,0 \%) \\
\text { Otra cirugía } 7(5,7 \%) \\
\text { Bilobectomía } 5(4,1 \%) \\
\text { Estereotomía } 2(1,6 \%)\end{array}$ \\
\hline ESTADIO & $\begin{array}{r}\text { In situ } 1(0,8 \%) \\
\text { Estadio IA } 11(9,0 \%) \\
\text { Estadio IB } 23(18,9 \%) \\
\text { Estadio IIB } 9(7,4 \%) \\
\text { Estadio IIIA } 12(9,8 \%) \\
\text { Estadio IIIB } 8(6,6 \%) \\
\text { Estadio IV } 3(2,5 \%) \\
\text { Metástasis } 26(21,3 \%)\end{array}$ \\
\hline ANTECEDENTES PERSONALES DE CÁNCER & $\begin{array}{r}\text { No antecedentes } 60(49,2 \%) \\
\text { Cáncer colon } 16(13,1 \%) \\
\text { Cáncer próstata } 9(7,4 \%) \\
\text { Osteosarcoma } 5(4,1 \%) \\
\text { Cáncer de mama } 3(2,5 \%)\end{array}$ \\
\hline FUMADOR & $\begin{array}{r}\text { No } 23(18,9 \%) \\
\text { Actualmente fuma } 18(14,8 \%) \\
\text { Fumador previo } 79(64,8 \%)\end{array}$ \\
\hline CONSUMO ALCOHOL & $\begin{array}{r}\text { No } 49(40,2 \%) \\
\text { Ocasional } 55(45,1 \%) \\
\text { Habitual } 7(5.7 \%)\end{array}$ \\
\hline ANTECEDENTES PSICOLÓGICOS & $\begin{array}{r}\text { No } 91(74,6 \%) \\
\text { Tr. del estado de ánimo } 12(9,8 \%) \\
\text { Tr. de ansiedad } 9(7,4 \%) \\
\text { Tr. de abuso de sustancias } 5(4,1 \%)\end{array}$ \\
\hline TRATAMIENTO PSICOFARMACOLÓGICO & $\begin{array}{r}\text { No } 86(70,5 \%) \\
\text { Antidepresivos } 9(7,4 \%) \\
\text { Ansiolíticos } 10(8,2 \%) \\
\text { Inductores del sueño } 5(4,1 \%) \\
\text { Ansiolíticos y antidepresivos } 1(0,8 \%)\end{array}$ \\
\hline
\end{tabular}

La ansiedad y depresión correlacionaron de manera positiva $(r=0,642 ; p=0,000)$. Calidad de vida global correlacionó negativamente con depresión $(r=-0,461$; $\mathrm{p}=0,000)$ y ansiedad $(\mathrm{r}=-0,304 ; \mathrm{p}=0,001)$.

Se detecta una correlación negativa entre depresión y todas las subescalas funcionales (ver tabla 5): funcionamiento físico ( $r=-0,393 ; p=0,000)$, rol $(r=-0,458$; $\mathrm{p}=0,000)$, cognitivo $(\mathrm{r}=-0,339, \mathrm{p}=0,000)$, emocional $(r=-0,610 ; p=0,000)$, y social $(r=-0,244 ; p=0,005)$. No existe correla- 
Tabla 3. Calidad de vida para la muestra prequirúrgica.

\begin{tabular}{|c|c|c|c|}
\hline & $\begin{array}{c}\text { Para la presente } \\
\text { investigación }(n=121) \\
\text { MEDIA (D.T) }\end{array}$ & $\begin{array}{c}\text { Para muestra europea* } \\
(n=1292) \\
\text { MEDIA(D.T) }\end{array}$ & $\begin{array}{c}\text { SIGN. } \\
\text { (bilateral) }\end{array}$ \\
\hline $\begin{array}{l}\text { ESCALA GLOBAL SALUD / } \\
\text { CALIDAD DE VIDA }\end{array}$ & $72,5(20,15)$ & $58,8(22,5)$ &, 000 \\
\hline \multicolumn{4}{|c|}{ ESCALAS FUNCIONALES } \\
\hline FÍSICA & $88,24(14,64)$ & $78,4(19,3)$ & 000 \\
\hline $\mathrm{ROL}$ & $88,64(20,06)$ & $60,7(33,1)$ & ,000 \\
\hline COGNITIVA & $89,4(15,92)$ & $84,0(21,1)$ & 000 \\
\hline EMOCIONAL & $75,47(19,01)$ & $68,1(24,2)$ & 001 \\
\hline SOCIAL & $89,7(19,42)$ & $73,6(28,9)$ & ,000 \\
\hline \multicolumn{4}{|c|}{ ESCALAS SINTOMÁTICAS } \\
\hline FATIGA & $18,59(19,97)$ & $40,7(27)$ & , 000 \\
\hline DOLOR & $13,76(19,22)$ & $29,7(30,3)$ & 000 \\
\hline NAÚSEAS Y VÓMITOS & $3,79(10,75)$ & $9,7(18,3)$ & , 000 \\
\hline DISNEA-C30**** & $11,81(23,72)$ & $38,5(31,7)$ &, 000 \\
\hline INSOMNIO & $25,76(30,51)$ & $32,4(32,7)$ & 024 \\
\hline APETITO & $10,30(22)$ & $27,9(33,5)$ & 000 \\
\hline ESTREÑIMIENTO & $12,12(21,99)$ & $17,4(27,9)$ & 013 \\
\hline DIARREA & $6,67(16,15)$ & $6,8(17,4)$ & 931 \\
\hline PROBLEMAS ECONÓMICOS & $6,67(17,95)$ & $12,8(25,8)$ & 001 \\
\hline \multicolumn{4}{|c|}{ SÍNTOMAS ESPECÍFICOS DE CÁNCER DE PULMÓN } \\
\hline DISNEA-LC13**** & $86,54(16,64)$ & & \\
\hline TOS & $30(25,92)$ & & \\
\hline HEMOPTISIS & $4,55(14,62)$ & & \\
\hline DOLOR EN LA BOCA & $6,67(17,37)$ & & \\
\hline DOLOR EN EL PECHO & $10,91(18,12)$ & & \\
\hline DOLOR BRAZO/HOMBRO & $11,82(22,39)$ & & \\
\hline DOLOR OTRAS PARTES & $16,97(25,84)$ & & \\
\hline DISFAGIA & $3,94(12,56)$ & & \\
\hline NEUROPATÍA & $16,36(25,84)$ & & \\
\hline ALOPECIA & $7,58(20,52)$ & & \\
\hline CONSUMO MEDICAMENTOS & $11,82(16,02)$ & & \\
\hline
\end{tabular}

* Scott, Fayers, Aaronson, Bottomley, de Graeff, Groenvold y EORTC Quality of Life Group (en línea) EORTC QLQ-C30. Reference values manual(44)

** No se ha encontrado ninguna investigación en español que aporte datos comparables con nuestra muestra, la única disponible es la de Arrarás, Martínez,Manterola, Salgado, Martínez, Vera, Illarramendi (2005) ${ }^{(45)}$ cuya muestra está compuesta por 97 pacientes con NSCLC pero en estadios muy avanzados (III y IV).

*** No se encontraron datos referenciales para los síntomas específicos de cáncer de pulmón medidos con el QLQ-LC13

**** Disnea-C30 hace referencia al síntoma de disnea medido por el cuestionario QLQ-C30 y Disnea-LC13 hace referencia al medido por el QLQ-LC13 
Tabla 4. Estado emocional en la muestra prequirúrgica.

\begin{tabular}{|l|c|c|}
\cline { 2 - 3 } \multicolumn{1}{c|}{} & DEPRESIÓN & ANSIEDAD \\
\hline Media (DT) & $4,36(3,62 \%)$ & $6,34(4,28 \%)$ \\
\hline Normal & $89(79,46 \%)$ & $76(67,86 \%)$ \\
\hline Subclínica & $18(16,07 \%)$ & $23(20,4 \%)$ \\
\hline Clínica & $5(4,46 \%)$ & $13(11,61 \%)$ \\
\hline
\end{tabular}

ción negativa entre depresión y las escalas sintomáticas. Correlación positiva con fatiga $(r=0,410 ; p=0,000)$, dolor $(r=0,227$; $\mathrm{p}=0,017)$ disnea-C30 $(r=0,248 ; \mathrm{p}=0,009)$, insomnio $(r=0,368 ; \quad p=0,000)$, apetito $(r=0,274 ; p=0,004)$, disnea-LC13 $(r=0,345$; $p=0,000)$, toser $(r=0,207 ; p=0,030)$, neuropatía $(r=0,202 ; p=0,034)$ y dolor en otras partes $(r=0,247 ; p=0,009)$.

Se encuentra una correlación negativa entre ansiedad y todas las subescalas funcionales (ver tabla 5): físico $(r=-0,221$; $p=0,021)$, rol $(r=-0,364 ; p=0,000)$, cognitivo $(r=-0,34 ; \quad p=0,000)$, emocional $(r=-0,805 ; p=0,000)$, y social $(r=-0,311$; $p=0,001)$. No hay correlación negativa entre ansiedad y las subescalas sintomáticas. Las correlaciones positivas se dan con fatiga $(r=0,323 ; p=0,001)$, dolor $(r=0,190 ; p=0,048)$, disnea-C30 $(r=0,23$; $p=0,016)$, insomnio $(r=0,441 ; p=0,000)$, apetito $(r=0,235 ; p=0,013)$, disnea-LC13 $(r=0,365 ; p=0,000)$ y dolor en otras partes $(r=0,254 ; p=0,008)$ y consumo de medicamentos $(r=0,22 ; p=0,022)$.

\section{Estrategias de afrontamiento (MAC)}

Únicamente disponemos de una medición post-operatoria, puesto que el estudio preoperatorio de los pacientes se inició antes de que se empezara a llevar a cabo la presente investigación.

Las estrategias de afrontamiento más utilizadas por los pacientes fueron en primer lugar el fatalismo seguido del espíritu de lucha. Las menos utilizadas fueron la indefensión y la preocupación ansiosa (ver tabla 6).
Las correlaciones entre las estrategias de afrontamiento muestran que el espíritu de lucha correlaciona negativamente con indefensión ( $r=-0,457 ; p=0,000)$. Preocupación ansiosa correlaciona positivamente con indefensión $(r=0,375 ; p=0,003)$ y con negación ( $r=0,292 ; p=0,023)$. El espíritu de lucha correlaciona negativamente con indefensión $(p=0,000)$.

En cuanto a las correlaciones entre estrategias de afrontamiento y calidad de vida podemos ver en la tabla 7 cuales mostraron correlaciones significativas.

Se obtienen correlaciones positivas entre depresión y preocupación ansiosa $(r=0,377 ; p=0,005)$. Ansiedad correlaciona positivamente con indefensión $(r=0,279$; $p=0,041)$ y con preocupación ansiosa $(r=0,394 ; p=0,003)$.

\section{Diferencias en calidad de vida y estado emocional en función del sexo y la intervención quirúrgica}

\section{En función del sexo}

Se realizó una comparación de medias entre las puntuaciones de los cuestionarios (QLQ-C30, QLQ-LC13 y HAD) y la variable sexo. Se aplicaron las pruebas de T de Student y U de Mann-Whitney. Al mismo tiempo, se calculó el tamaño del efecto mediante la prueba $d$ de Cohen.

No hay diferencias en calidad de vida global entre hombres y mujeres pero sí se encontraron en la escala funcional física $(p=0,03)$, obteniendo los hombres una media de $91,67(\mathrm{DT}=10,67)$ y las mu- 
Tabla 5. Correlaciones significativas entre la calidad de vida y estado emocional

\begin{tabular}{|c|c|c|}
\hline & DEPRESIÓN & ANSIEDAD \\
\hline \multirow[t]{2}{*}{ CALIDAD DE VIDA GLOBAL } &,- 461 &,- 304 \\
\hline & $\mathrm{p}=, 000$ & $\mathrm{p}=, 001$ \\
\hline \multirow[t]{2}{*}{ FUNCIONAMIENTO FÍSICO } &,- 393 &,- 221 \\
\hline & $p=, 000$ & $\mathrm{p}=, 021$ \\
\hline \multirow[t]{2}{*}{ FUNCIONAMIENTO DE ROL } &,- 458 &,- 364 \\
\hline & $\mathrm{p}=, 000$ & $\mathrm{p}=, 000$ \\
\hline \multirow[t]{2}{*}{ FUNCIONAMIENTO EMOCIONAL } &,- 610 &,- 805 \\
\hline & $p=, 000$ & $\mathrm{p}=, 000$ \\
\hline \multirow[t]{2}{*}{ FUNCIONAMIENTO COGNITIVO } &,- 339 &,- 340 \\
\hline & $\mathrm{p}=, 000$ & $\mathrm{p}=, 000$ \\
\hline \multirow[t]{2}{*}{ FUNCIONAMIENTO SOCIAL } &,- 244 &,- 311 \\
\hline & $p=, 005$ & $p=, 001$ \\
\hline \multirow[t]{2}{*}{ FATIGA } &, 410 & ,323 \\
\hline & $p=, 000$ & $p=, 001$ \\
\hline \multirow{2}{*}{ DOLOR } &, 227 &, 190 \\
\hline & $p=, 017$ & $p=, 048$ \\
\hline \multirow[t]{2}{*}{ DISNEA-C30 } &, 248 & ,230 \\
\hline & $p=, 009$ & $p=, 016$ \\
\hline \multirow[t]{2}{*}{ INSOMNIO } & ,368 &, 441 \\
\hline & $p=, 000$ & $p=, 000$ \\
\hline \multirow[t]{2}{*}{ APETITO } &, 274 & ,235 \\
\hline & $\mathrm{p}=, 004$ & $p=, 013$ \\
\hline \multirow[t]{2}{*}{ DISNEA-LC13 } & ,345 & ,365 \\
\hline & $p=, 000$ & $p=, 000$ \\
\hline \multirow[t]{2}{*}{ TOSER } & ,207 & \\
\hline & $\mathrm{p}=, 030$ & \\
\hline \multirow{2}{*}{ NEUROPATÍA } & ,202 & \\
\hline & $\mathrm{p}=, 034$ & \\
\hline \multirow[t]{2}{*}{ DOLOR EN OTRAS PARTES } &, 247 &, 254 \\
\hline & $p=, 009$ & $p=, 008$ \\
\hline \multirow[t]{2}{*}{ CONSUMO DE MEDICAMENTOS } & & ,218 \\
\hline & & $\mathrm{p}=, 022$ \\
\hline
\end{tabular}


Tabla 6. Estrategias de afrontamiento postquirúrgicas.

\begin{tabular}{|c|c|c|c|c|}
\hline $\mathrm{N}=65$ & Media & D.T & Frecuencia & $\%$ \\
\hline Espíritu de lucha & 51 & 7 & 43 & 70,49 \\
\hline Indefensión & 9,02 & 3,63 & 9 & 14,52 \\
\hline Preocupación ansiosa & 20 & 4,71 & 5 & 8,06 \\
\hline Fatalismo & 24,77 & 3,83 & 43 & 71,67 \\
\hline Negación & 2,25 & 1,39 & 27 & 44,3 \\
\hline
\end{tabular}

Tabla 7. Correlaciones significativa entre calidad de vida y estrategias de afrontamiento.

\begin{tabular}{|c|c|c|c|c|}
\hline & Espíritu de lucha & Indefensión & Preocupación ansiosa & Fatalismo \\
\hline CV Global & $\begin{array}{c}, 408 \\
p=, 002\end{array}$ & & & \\
\hline F. Físico & & & $\begin{array}{l}-, 287 \\
p=, 035\end{array}$ & \\
\hline F. Rol & $\begin{array}{l}, 326 \\
p=, 016\end{array}$ & $\begin{array}{l}-, 347 \\
p=, 01\end{array}$ & $\begin{array}{l}-, 341 \\
p=12\end{array}$ & $\begin{array}{c}, 409 \\
p=, 002\end{array}$ \\
\hline F. Emocional & & $\begin{array}{l}-, 307 \\
p=, 027\end{array}$ & $\begin{array}{l}-, 374 \\
p=, 06\end{array}$ & \\
\hline F. Cognitivo & $\begin{array}{l}, 272\left(^{*}\right) \\
p=47\end{array}$ & $\begin{array}{c}-, 270 \\
p=, 048\end{array}$ & & \\
\hline F. Social & $\begin{array}{l}, 359 \\
p=, 08\end{array}$ & & & \\
\hline Fatiga & $\begin{array}{l}-, 311\left(^{*}\right) \\
p=, 022\end{array}$ & & & \\
\hline Disnea C-30 & $\begin{array}{c}-, 460(* *) \\
p=00\end{array}$ & $\begin{array}{c}, 400 \\
p=, 003\end{array}$ & $\begin{array}{c}, 412 \\
p=, 002\end{array}$ & $\begin{array}{l}-, 306 \\
p=, 026\end{array}$ \\
\hline Insomnio & $\begin{array}{l}-, 339 \\
p=, 012\end{array}$ & & $\begin{array}{c}, 346 \\
p=, 010\end{array}$ & \\
\hline Problemas económicos & & & & $\begin{array}{l}-, 297 \\
p=, 031\end{array}$ \\
\hline Disnea LC13 & $\begin{array}{l}-, 321\left(^{*}\right) \\
\mathrm{p}=, 018\end{array}$ & & $\begin{array}{c}, 284 \\
p=, 037\end{array}$ & \\
\hline Toser & & & & $\begin{array}{l}-, 285 \\
p=, 033\end{array}$ \\
\hline
\end{tabular}


jeres 85,96 (DT= 16,469). En las escalas sintomáticas se encuentran diferencias en disnea-C30 $(p=0,018)$ donde los hombres presentan mayores problemas con una media de 15,66 (DT= 28,18) que las mujeres: 6,06 (DT=13), mientras que las mujeres puntúan más en neuropatía (media= $23,48, \mathrm{DT}=30,99$ ) que los hombres (media $=11,62, \mathrm{DT}=20,67, \mathrm{p}=0,029$ ).

Las mujeres presentaron más ansiedad que los hombres $(p=0,001)$ pero esta diferencia se detecta habitualmente.

No se obtuvieron diferencias estadísticas significativas en la variable sintomatología depresiva en función de la variable sexo y los tamaños del efecto son pequeños.

\section{En función de intervención quirúrgica}

Se llevo a cabo la prueba de KruskalWallis para comprobar las diferencias entre los tres tipos de intervención quirúrgica mas frecuentes: resección, lobectomía y neumonectomía. Se calculó el tamaño del efecto mediante la prueba $d$ de Cohen. Las diferencias en calidad de vida según intervención quirúrgica se exponen en la tabla 8.

No hubo diferencias significativas en las puntuaciones de calidad de vida global ni en las escalas funcionales según el tipo de intervención quirúrgica.

En las escalas sintomáticas se encuentraron diferencias en dolor en el pecho $(p=0,011)$, los neumonectomizados presentaron mayor sintomatología que los reseccionados según la prueba $U$ de MannWhitne $(p=0,026)$. En cuanto a la variable sangrar hubo diferencias entre los distintos tipos de cirugías, siendo los neumonectomizados los que presentaron mayor sintomatología y con ello mayor afectación que los reseccionados $(p=0,014)$ y los lobectomizados $(p=0,004)$, según al prueba de $U$ de Mann-Whitney.

Cabe mencionar que el mayor tamaño del efecto $(d \geq 0,80)$ se encuentra en las variables funcionamiento de rol $(d=0,80$ entre resección segmentaria y neumonectomía; $d=0,97$ entre lobectomía y neumonectomía). Dolor ( $d=-0,97$ entre resección segmentaria y neumonectomía), toser $(d=-1,05$ entre resección segmentaria y neumonectomía), sangrar $(d=-0,86$ entre resección segmentaria y neumonectomía; $d=-1,12$ entre lobectomía y neumonectomía). Dolor en el pecho ( $d=-1,16$ entre resección segmentaria y neumonectomía). Dolor en otras partes $(d=-0,86$ entre resección segmentaria y neumonectomía; $d=-0,92$ entre lobectomía y neumonectomía).

No se encontraron diferencias estadísticamente significativas en ansiedad y depresión según el tipo de intervención quirúrgica, y los tamaños del efecto eran pequeños.

\section{CONCLUSIÓN}

La calidad de vida global del paciente con cáncer de pulmón que va a ser intervenido fue alta $(72,5)$ superando la media para la muestra europea $(58,8)$.

Las diferencias en calidad de vida según la variable sexo únicamente se encuentraron en funcionamiento físico, disnea y neuropatía. Según el tipo de cirugía, los pacientes que iban a ser neumonectomizados tuvieron mayor sintomatología en cuanto al sangrar, dolor en el pecho y en otras partes, y otros déficits en calidad de vida.

La disnea fue el principal síntoma que se asoció a déficit en calidad de vida, depresión y ansiedad, además de otros síntomas físicos como fatiga y dolor.

A pesar del diagnóstico de enfermedad maligna, el $79,46 \%$ no presentó sintomatología depresiva. El 20,53\% (que equivale a 23 pacientes) presentó sintomatología depresiva $(16,07$ \%subclínica y 4,46\% clínica). 1/3 de los pacientes presentó sintomatología ansiosa $(20,54 \%$ subclínica y 11,61\% clínica).

Las estrategias de afrontamiento más 
Tabla 8. Diferencias en calidad de vida según intervención quirúrgica. Medias, desviaciones típicas, significación de la prueba de Kruskall-Wallis, y tamaño del efecto por pares

\begin{tabular}{|c|c|c|c|c|c|c|c|}
\hline & $\begin{array}{c}\text { RESECCIÓN } \\
\text { SEGMENT. } \\
(\mathrm{n}=48) \\
\text { Media (DT) }\end{array}$ & $\begin{array}{c}\text { LOBECTOMÍA } \\
(\mathrm{n}=43) \\
\text { Media (DT) }\end{array}$ & $\begin{array}{c}\text { NEUMONECTOMÍA } \\
(\mathrm{n}=11) \\
\text { Media (DT) }\end{array}$ & $\mathrm{p}$ & $\begin{array}{c}\text { TAMAÑO } \\
\text { DEL } \\
\text { EFECTO } \\
\text { RS-LOB }\end{array}$ & $\begin{array}{c}\text { TAMAÑO } \\
\text { DEL } \\
\text { EFECTO } \\
\text { RS-NEU }\end{array}$ & $\begin{array}{c}\text { TAMAÑO } \\
\text { DEL } \\
\text { EFECTO } \\
\text { LOB-NEU }\end{array}$ \\
\hline CV Global & $73,73(19,95)$ & $72,75(18,80)$ & $68,75(33,56)$ & ,89 & $-0,25$ & 0,22 & 0,18 \\
\hline F. Físico & $88,7(14,73)$ & $88,11(16,64)$ & $80(11,82)$ & ,11 & $-0,10$ & 0,61 & 0,51 \\
\hline F. Rol & $89,49(19,36)$ & $91,44(15,53)$ & $70,83(36,46)$ & ,12 & 0,62 & 0,80 & 0,97 \\
\hline F. Emocional & $78,03(16,96)$ & $77,48(19,43)$ & $72,62(17,16)$ &, 62 & 0,14 & 0,32 & 0,26 \\
\hline F. Cognitivo & $88,04(16,35)$ & $91,89(14,5)$ & $85,42(10,68)$ & ,13 & $-0,25$ & 0,17 & 0,47 \\
\hline F. Social & $88,41(20,45)$ & $90,54(20,62)$ & $83,33(25,2)$ &, 05 & $-0,14$ & 0,24 & 0,33 \\
\hline Fatiga & $17,87(17,76)$ & $16,22(21,21)$ & $22,22(22,22)$ &, 53 & $-0,04$ & $-0,23$ & $-0,28$ \\
\hline $\begin{array}{l}\text { Náuseas y } \\
\text { vómitos }\end{array}$ & $3,26(8,33)$ & $2,25(5,78)$ & ,000 (,000) &, 50 & 0,07 & 0,43 & 0,43 \\
\hline Dolor & $11,49(16,6)$ & $25,69(18,06)$ & $31,25(32,66)$ & ,22 & 0,01 & $-0,97$ & $-0,26$ \\
\hline Disnea-C30 & $9,42(22,95)$ & $12,61(24,03)$ & $20,83(30,54)$ &, 46 & $-0,29$ & $-0,47$ & $-0,32$ \\
\hline Insomnio & $23,91(31,16)$ & $25,23(28,77)$ & $33,33(35,63)$ & ,68 & 0,00 & $-0,29$ & $-0,27$ \\
\hline Apetito & $8,7(19,16)$ & $7,21(20,99)$ & $25(34,50)$ & ,07 & 0,14 & $-0,72$ & $-0,74$ \\
\hline Estreñimiento & $10,14(19,71)$ & $9,91(20,59)$ & $16,67(25,2)$ & ,68 & $-0,23$ & $-0,31$ & $-0,31$ \\
\hline Diarrea & $4,35(13,35)$ & $9(18,67)$ & $8,33(15,43)$ &, 37 & 0,23 & $-0,29$ & 0,04 \\
\hline $\begin{array}{l}\text { Problemas } \\
\text { económicos }\end{array}$ & $7,24(19,77)$ & $7,21(17,80)$ & $8,33(23,57)$ & ,98 & $-0,24$ & $-0,05$ & $-0,06$ \\
\hline Disnea-LC13 & $11,35(13,76)$ & $13,51(17,59)$ & $14,29(13,93)$ &, 30 & $-0,14$ & $-0,21$ & $-0,05$ \\
\hline Toser & $23,19(20,93)$ & $28,83(27,4)$ & $45,83(24,8)$ &, 064 & $-0,01$ & $-1,05$ & $-0,63$ \\
\hline Sangrar & $4,35(13,35)$ & $1,80(7,64)$ & $20,83(35,36)$ & ,028 & $-0,20$ & $-0,86$ & $-1,12$ \\
\hline Dolor boca & $4,34(13,35)$ & $8,11(18,27)$ & ,000 $(, 000)$ & ,28 & $-0,36$ & 0,36 & 0,49 \\
\hline Disfagia & $2,17(8,32)$ & $4,50(13,97)$ & $4,17(11,79)$ & 0,72 & $-0,03$ & $-0,22$ & 0,02 \\
\hline Neuropatía & $15,94(24,08)$ & $16,21(27,91)$ & $4,17(11,79)$ &, 40 & 0,07 & 0,53 & 0,47 \\
\hline Alopecia & $7,25(15,58)$ & $11,71(28,56)$ & ,0000 (,00000) &, 42 & $-0,14$ & 0,51 & 0,46 \\
\hline Dolor pecho & $5,8(14,58)$ & $11,71(17,94)$ & $25(23,57)$ & ,011 & $-0,25$ & $-1,16$ & $-0,69$ \\
\hline Dolor brazo & $10,14(18,42)$ & $10,81(24,91)$ & $4,17(11,79)$ & ,07 & $-0,10$ & 0,34 & 0,29 \\
\hline $\begin{array}{l}\text { Dolor otras } \\
\text { partes }\end{array}$ & $15,22(24,04)$ & $13,51(24,16)$ & $37,5(33,03)$ & ,043 & 0,43 & $-0,86$ & $-0,92$ \\
\hline $\begin{array}{l}\text { Consumo } \\
\text { medicamentos }\end{array}$ & $8,7(14,8)$ & $10,81(15,82)$ & $20,83(17,25)$ & 0,12 & 0,14 & $-0,79$ & $-0,62$ \\
\hline
\end{tabular}


utilizadas por los pacientes con cáncer de pulmón fueron en primer lugar el fatalismo $(71,67 \%)$ seguido del espíritu de lucha $(70,49 \%)$. Es importante medir las estrategias de afrontamiento ya que se asociaron con las escalas funcionales $y$ sintomáticas de calidad de vida y estado emocional.

A modo preventivo, se debería evaluar a todos los pacientes oncológicos que van a ser sometidos a intervención quirúrgica y así poder seleccionar aquellos pacientes de alto riesgo. Los pacientes a los que se les practica la neumoctomía presentan mayores problemas de calidad de vida que lobectomizados y reseccionados. Es primordial no sólo atender a la parte física-médica del paciente sino que también es igual de necesario complementarlo con apoyo psicológico, social y espiritual. Conocer cuales son las necesidades de los pacientes de cáncer de pulmón nos puede ayudar a atenerles de manera integral. Como decía Cassell (1982) "Los que sufren no son los cuerpos, son las personas".

\section{Limitaciones y directrices futuras}

Las limitaciones de este estudio fueron a nivel muestral (imposibilidad de generalizar los resultados a otros tipos de cáncer de pulmón) y temporal (únicamente una evaluación previa a la cirugía).

Las directrices futuras van encaminadas a introducir un grupo control a modo de comparación, seguir con un estudio longitudinal (post-operatorio y seguimiento) y diseñar intervenciones psicológicas encaminadas a reducir y/o controlar la sintomatología ansiosa y depresiva.

\section{REFERENCIAS BIBLIOGRÁFICAS}

1. Calzas J, Barneto I, Sánchez M. Cáncer de Pulmón. Cuestiones más frecuentes. Madrid: Grupo Editorial Entheos; 2007. p. 24-6.
2. Aaronson NK, Ahmedzai S, Bergman B, Bullinger M, Cull A, Duez M. The European Organization for Research and Treatment of Cancer QLQ-C30: A quality of life instrument for use in international clinical trials. J Natl Cancer Inst 1993; 85: 365-76. Doi: 10.1093/jnci/85.5.365>

3. Schag CA, Ganz PA, Wing DS, Sim MS, Lee JJ. Quality of life in adult survivors of lung, colon and prostate cancer. Qual Life Res. 1994; 3(2):127-1. Doi: 10.1007/ BF00435256.

4. Kaasa S, Mastekaasa A, Lund E. Prognostic factors for patients with inoperable nonsmall cell lung cancer, limited disease. Radiother Oncol 1989; 15(3): 235-42. Doi:10.1016/0167-8140(89)90091-1.

5. Ganz PA, Lee JJ, Siau J. Quality of life assessment. An independent prognostic variable for survival in lung cancer. Cancer 1991:67(12): 3131-5. Doi: 10.1002/10970142(19910615)67:12<3131: :AID-CNCR 2820671232>3.0.CO;2-4

6. Degner LF, Sloan JA. Symptom distress in newly diagnosed ambulatory cancer patients and as a predictor of survival in lung cancer. J Pain Symptom Manage 1995; 10(6): 423-31. Doi: 10.1016/08853924(95)00056-5

7. Montazeri A, Milroy R, Gillis CR, McEwen J. Quality of life: perception of lung cancer patients. Eur J Cancer 1996; 32A (13): 2284-9. Doi:10.1016/S09598049(96)00288-2

8. Buccheri G. Depressive reactions to lung cancer are common and often followed by a poor outcome. Eur Respir J 1998; 11: 173-178. Doi: 10.1183/09031936.98.11010173

9. Montazeri A, Gillis CR, McEwen J. Quality of life in patients with lung cancer: A review of literature from 1970 to 1995 . Chest 1998; 113: 467-81. Doi: 10.1378/ chest.113.2.467.

10. Langendijk H, Aaronson N, Jong J, Ten Velde G, Muller M, Wouters M. The prognostic impact of quality of life assessed with the 
EORTC QLQ-C30 in inoperable non-small cell lung carcinoma treated with radiotherapy. Radiother Oncol 2000; 55(1): 19-25. Di:10.1016/S0167-8140(00)00158-4

11. Zabora J, Brintzenhofeszoc K, Curbow B, Hooker C, Piantadosi S. The prevalence of psychological distress by cancer site. Psychooncology 2001; 10: 19-28. Doi: 10.1002/1099-1611(200101/02)10:1<19: :AID-PON501>3.0.CO;2-6

12. Barlési F, Doddoli C, Loundou A, Pillet E, Thomas P, Auquier P. Preoperative psychological global well being index predicts postoperative quality of life for patients with non-small cell lung cancer managed with thoracic surgery. Eur J Cardiothorac Surg 2006; 30: 548-53. Doi:10.1016/j. ejcts.2006.05.032.

13. Brunelli A, Socci L, Refai M, Salati M, Xiumé F, Sabbatini A. Quality of life before and after major lung resection for lung cancer: A prospective follow-up analysis. Ann Thorac Surg 2007; 84: 410-6. Doi: 10.1016/j.athoracsur.2007.04.019.

14. Fuentes PA. Pneumonectomy: historical perspective and prospective insight. Eur J Cardiothorac Surg 2003; 23: 439-45. Doi: 10.1016/S1010-7940(03)00117-9.

15. Paull DE, Thomas M, Meade G, Updyke GM, Arocho MA, Chin HW, et al. Determinants of quality of life in patients following pulmonary resection for lung cancer. Am J Surg 2006; 192: 565-71. Doi:10.1016/j. amjsurg.2006.08.019.

16. Folkman S, Lazarus RS. If it changes, it must be a process: A study of emotion and coping during three stages of a collage examination. J Pers Soc Psychol 1984; 50: 992-1033. Doi:10.1037/00223514.48.1.150.

17. Cruzado JA, Olivares ME. Tristeza y depresión en el cáncer. En Palmero, F. y Fernández-Abascal EG, editores. Emociones y adaptación. Barcelona: Ariel Psicología. 1998: p.163-89

18. Hopwood P, Stephens R. Depression in patients with lung cancer: prevalence and risk factors derived from quality of life data. J Clin Oncol 2000; 18(4): 893-903.

19. Uchitomi Y, Mikami I, Kugaya A, Akizuki N, Nagai K, Nishiwaki Y et al. Depression after successful treatment for nonsmall cell lung carcinoma: A 3-month follow-up. Cancer 2000; 89: 1172-9. Doi: 10.1002/10970142(20000901)89:5<1172: :AID-CNCR $27>3.0 . \mathrm{CO} ; 2-\mathrm{U}$

20. Oh S, Miyamoto H, Yamazaki A, Fukai $\mathrm{R}$, Shiomi K, Sonobe S et al. Prospective analysis of depression and psychological distress before and after surgical resection of lung cancer. Gen Thorac Cardiovasc Surg 2007; 55:119-124. Doi: 10.1007/ s11748-006-0084-4.

21. Maric DM, Jovanovic DM, Golubicic IV, Nagorni-Obradovic LJ, Stojsic JM, Pekmezovic TD. Psychological well-being in advanced NSCLC patients in Serbia: Impact of sociodemographic and clinical factors. Neoplasma 2010; 57 (1): 1-7. Doi:10.4149/neo_2010_01_001

22. Carrol BT, Kathol RG, Noyes R, Wald TG, Clamon $\mathrm{GH}$. Screening for depression and anxiety in cancer patients using the Hospital Anxiety and Depression Scale. Gen Hosp Psychiatry 1993; 15(2): 69-74. Doi:10.1016/0163-8343(93)90099-A.

23. Aass N, Dahl AA, Moe TJ. Prevalence of anxiety and depression in cancer patients seen at the Norwegian Radium Hospital. Eur J Cancer 1997; 33: 1597-604. Doi:10.1016/S0959-8049(97)00054-3.

24. Liao YC, Liao WY, Shun SC, Yu CJ, Yang PC, Lai YH. Symptoms, psychological distress and supportive care needs in lung cancer patients. Support Care Cancer 2011; 19 (11): 1743-51. Doi: 10.1007/s00520-0101014-7

25. Sanders SI, Bantum EO, Owen JE, Thornton AA, Stanton AL. Supportive care needs in patients with lung cancer. Psychooncology 2010; 19 (5): 480-9. Doi: 10.1002/ pon. 1577

26. Carlsen K, Bonde-Jensen A, Jacobsen E, Krasnik M, Johansen C. Psychologi- 
cal aspects of lung cancer. Lung Cancer 2005; 47: 293-300. Doi:10.1016/j.lungcan.2004.08.002.

27. Graves K, Arnold S, Love C, Kirsh K, Moore $\mathrm{P}$, Passik S. Distress screening in a multidisciplinary lung cancer clinic: prevalence and predictors of clinically significant distress. Lung Cancer 2007; 55:215-224. Doi:10.1016/j.lungcan.2006.10.001.

28. Cody M, Nichols Brennan C, Armes J, Wilson P, Slevin M. Psychosocial factors and lung cancer prognosis. Psychooncology 1994; 3:141.

29. Sarna L, Cooley ME, Brown JK, Chernecky C, Padilla G, Danao L, Chakravarty D, Elashoff D. Women with lung cancer: quality of life after tohoracotomy: A 6-month prospective study. Cancer Nur 2010; 33(2): 85-92. Doi: 10.1097/ NCC.0b013e3181be5e51

30. Martín-Ortiz JD, Sánchez MJ, Sierra C. Evaluación de calidad de vida en pacientes con cáncer: una revisión. Rev Colomb Psicol 2005; 14: 34-45.

31. Buchanan D, Milroy R, Baker L, Thompson $M$, Levack P. Perceptions of anxiety in lung cancer patients and their support network. Support Care Cancer 2009; 18:29-36. Doi:10.1007/s00520-009-0626-2.

32. Watson $M$, Greer $S$, Young J, Inayat Q, Burgess C, Robertson B. Development of a questionnaire measure of adjustment to cancer: The MAC scale. Psychol Med 1988; 18, 203-9. Doi:10.1017/ S0033291700002026

33. Moorey S, Greer S. Adjuvant Psychological Therapy: A cognitive behavioural treatment for patients with cancer. Behav Psychother 1989: 17 (2): 177-190. Doi: 10.1002/ pon.2960030108

34. Schnoll RA, Harlow LL, Brandt U, Stolbach LL. Using two factor structures of the mental adjustment to cancer (MAC) scale for assessing adaptation to breast cancer. Psychooncology 1998; 7(5): 424-35. Doi: 10.1002/ (SICI)1099-1611(1998090)7:5<424::AIDPON322>3.0.CO;2-2.
35. Camacho S. El Cáncer de Pulmón. En Die Trill M, editor. Psicooncología. Madrid: Ediciones Ades 2003. p: 207-24.

36. Vos MS, Putter $\mathrm{H}$, van Houwelingen $\mathrm{HC}$, de Haes HC. Denial and social and emotional outcomes in lung cancer patients: the protective effect of denial. Lung Cancer 2011; 72 (1): 119-24. Doi: 10.1016/j. lungcan.2010.07.007

37. Zigmond AS, Snaith RP. The Hospital Anxiety and Depression Scale. Acta Psychiatr Scand 1983; 67:361-8. Doi:10.1111/j.1600-0447.1983.tb09716.x

38. Caro I, Ibánez I. La escala Hospitalaria de ansiedad y depresión. Bol Psicol 1992; 36: 43-69.

39. Arrarás JI, Illarramendi JJ, Valerdi JJ. El cuestionario de calidad de vida para cáncer de la EORTC, QLQ-C30. Estudio estadístico de validación con una muestra española. Rev Psicol Salud 1995; (1): 13-34

40. Bergman B, Aaronson NK, Ahmedzai S, Kaasa S, Sullivan M. The EORTC QLQLC13: A modular supplement to the EORTC core quality of life questionnaire (QLQ-C30) for use in lung cancer clinical trials. Eur J Cancer 1994; 30 (5): 635-42. Doi:10.1016/0959-8049(94)90535-5

41. Arrarás JI, Prujá E, Marcos $M$, Tejedor M, Illarramendi JJ, Vera R et al. El cuestionario de calidad de vida para cáncer de pulmón de la EORTC QLQ-C13. Estudio de validación para nuestro país. Oncologia 2000; 23 (3): 127-34.

42. Watson $M$, Greer $S$, Young J, Inayat Q, Burgess C, Robertson B. Development of a questionnaire measure of adjustment to cancer: The MAC scale. Psychol Med 1988; 18: 203-9. Doi:10.1017/ S0033291700002026

43. Ferrero J, Barreto MP, Toledo M. Mental adjustment to cancer and quality of life in breast cancer patients: An exploratoly study. Psychooncology 1994; 3: 3-32. Doi:10.1002/pon.2960030309

44. Scott N, Fayers P, Aaronson N, Bottomley A, de Graeff A, Groenvold M et al. EORTC 
QLQ-C30. Reference values. Brussels: EORTC, 2008.

45. Arrarás JI, Martinez M, Manterola A, Salgado E, Martínez E, Vera R et al. Quality of life assessment in Spanish lung cancer patients by the EORTC questionnaires. Oncología 2005; 28: 174-82. 